\title{
Catalytic applications of aminopropylated mesoporous silica prepared by a template-free route in flavanones synthesis
}

\author{
Xueguang Wang, Yao-Hung Tseng, Jerry C.C. Chan, Soofin Cheng* \\ Department of Chemistry, National Taiwan University, Taipei 106, Taiwan
}

Received 23 February 2005; revised 7 April 2005; accepted 8 April 2005

Available online 6 June 2005

\begin{abstract}
Aminopropylated silica containing mesopores was prepared by a simple sol-gel process with tetraethyl orthosilicate (TEOS) and aminopropyltriethoxysilane (APTES) under strong acidic condition. The materials were characterized by $\mathrm{N}_{2}$ sorption, TGA, FTIR, and solid-state NMR measurements. It was proved that the amount of APTES in the initial mixture and the preparation process had a great influence on the textural properties of the hybrid organic-inorganic materials. The samples prepared by TEOS prehydrolysis and an appropriate amount of APTES (equal to or less than $10 \mathrm{~mol} \%$ ) contained mesoporous structures of narrow pore size distribution and possessed a larger surface area and pore volume than that prepared without TEOS prehydrolysis. The materials were used as catalysts in the Claisen-Schmidt condensation between substituted benzaldehydes and substituted $2^{\prime}$-hydroxyacetophenones and the subsequent isomerization of the $2^{\prime}$-hydroxychalcone intermediates in the liquid phase. The results showed that the presence of an appropriate amount of aminopropyl groups and narrow distributed mesopores was important for good performance of the catalysts. Good catalytic activities and very high selectivities for flavanones were obtained in solvent-free reactions. The influence of the substituting groups in the aromatic rings of benzaldehyde and $2^{\prime}$-hydroxyacetophenone was investigated under solvent-free conditions. The influence of electron-donating and electron-withdrawing groups at the para position of benzaldehyde was contrary to the results obtained over other base catalysts. The possible reaction mechanism involving the formation of imine intermediates was suggested for the synthesis of flavanone over aminopropylated silica.
\end{abstract}

(C) 2005 Elsevier Inc. All rights reserved.

Keywords: Aminopropylated silica; Mesoporous; Template-free; Prehydrolysis; Claisen-Schmidt condensation; Base catalyst; Chalcone; Flavanone

\section{Introduction}

Flavonoids are a ubiquitous group of polyphenolic substances that are present in most plants, preserving the health of plants against infections and parasites. They are used in numerous pharmacological applications with antimalarial [1], anticancer [2], anti-inflammatory [3], cytotoxic [4], antibacterial [5], and anti-AIDS [6] activities. Traditionally, flavonones were synthesized via the Claisen-Schmidt condensation between substituted benzaldehydes and substituted 2 '-hydroxyacetopheones and the subsequent isomerization of the 2 -hydroxychalcone intermediates in basic or acidic media under homogeneous conditions (Scheme 1)

\footnotetext{
* Corresponding author. Fax: +886223636359.

E-mail address: chem1031@ntu.edu.tw (S. Cheng).
}

[7-9]. Environmental regulations and process safety are driving the industry to develop new strategies to replace liquid acid or base processes.

Heterogeneous catalysis is widely accepted as an environmentally friendly alternative for the synthesis of fine chemicals and pharmaceuticals, since the use of heterogeneous catalytic processes not only allows easier separation, recovery, and recycling of the catalyst from the reaction mixture, but also gives better selectivity than homogeneous processes for many bimolecular reactions [10-12]. In the past decade, various solid catalysts have been applied to flavonoid synthesis, such as magnesium oxide [12], alumina [13], barium hydroxides [14,15], hydrotalcites [16-18], and natural phosphates modified with $\mathrm{NaNO}_{3}$ or KF $[19,20]$. However, most of them require rather complicated preparation of the catalysts or the use of expensive toxic solvents to facilitate heat 
<smiles>[R]c1ccc(C=O)cc1C(=O)/C=C/c1ccc(C(C)(C)C)c(O)c1</smiles>

1<smiles>[R]c1ccc(/C=C/C(=O)c2cc([R])ccc2O)cc1</smiles>

3
2

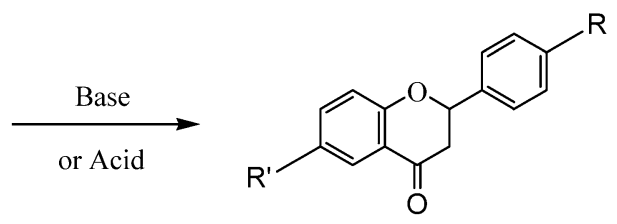

Scheme 1. (i) Claisen-Schmidt condensation and (ii) isomerization of $2^{\prime}$-hydroxychalcone intermediates.

and mass transfer in liquid-phase reaction systems [12]. As a clean route for organic synthesis, the ultimate goal of many heterogeneous catalytic processes is to eliminate the use of solvents.

Recently amino- or diamino-modified porous silicas were reported to be effective base catalysts for Knoevenagel condensations, Michael additions, and the nitroaldol reactions [21-24]. Here we report our recent findings on the efficient synthesis of flavanones by the ClaisenSchmidt condensation reaction under solvent-free conditions catalyzed by aminopropylated porous silica materials. The materials were prepared through a simple cocondensation method without the addition of any poredirecting agents. The catalytic efficiency of the aminopropylated silica is discussed for the Claisen-Schmidt condensation between substituted benzaldehydes and substituted $2^{\prime}$-hydroxyacetopheones and the subsequent isomerization of the $2^{\prime}$-hydroxychalcone intermediates in the absence of solvent.

\section{Experimental}

\subsection{Chemicals and catalyst synthesis}

All chemical reagents and solvents were purchased from Acros and used without further purification.

The aminopropylated silicas were prepared by the solgel process of tetraethoxysilane (TEOS) and aminopropyltriethoxysilane (APTES) under acid conditions. In the typical synthesis, $8 \mathrm{~g}$ of TEOS was first added to an opening beaker with $125 \mathrm{~g}$ of $2.0 \mathrm{M} \mathrm{HCl}$ solution and hydrolyzed at $40^{\circ} \mathrm{C}$ under stirring for $20 \mathrm{~h}$. When the mixture became a slightly viscous solution, the appropriate amount of APTES was slowly added, and the mixture was stirred at the same temperature until the water evaporated. The obtained dried solids were finely ground and then treated with a $0.2 \mathrm{M}$ methanol solution of tetramethylammonium hydroxide (TMAOH) for $20 \mathrm{~min}$ to neutralize the protonated amino groups (1.0 $\mathrm{g}$ of the gel per $50 \mathrm{ml}$ of solution). Finally, the material in powder form was filtered, washed several times with methanol, and dried at $100^{\circ} \mathrm{C}$. The resultant samples are designated Cat- $x$-P, where $\mathrm{P}$ denotes prehydrolysis of TEOS and $x$ is the molar percentage of APTES/(TEOS + APTES). For example, Cat-10-P represents the sample prepared with TEOS prehydrolysis and APTES $/($ TEOS + APTES $)=10 \mathrm{~mol} \%$. For comparison, the sample denoted Cat-10, containing 10 mol\% APTES, was prepared in a similar way without TEOS prehydrolysis. In other words, TEOS and APTES were added to the acid simultaneously. The other sample, denoted SBA-NH $\mathrm{N}_{2}$, was the mesoporous SBA-15 silica material containing $10 \mathrm{~mol} \%$ APTES, prepared according to the procedures reported in Ref. [24], with Pluronic 123 as the pore-directing agent, and by hydrothermal reaction at $90^{\circ} \mathrm{C}$ under static conditions for $24 \mathrm{~h}$. The solid product recovered by filtration and drying at room temperature overnight was refluxed in $95 \%$ ethanol for $24 \mathrm{~h}$ to remove the organic template. Finally, the material was treated with a $0.2 \mathrm{M}$ methanol solution of TMAOH for $20 \mathrm{~min}$ before it was used as the catalyst.

\subsection{Catalyst characterization}

$\mathrm{N}_{2}$ adsorption-desorption isotherms were measured with a Micromeritics Tristar 3000 at liquid nitrogen temperature. Before the measurements, the samples were degassed at $100{ }^{\circ} \mathrm{C}$ for $12 \mathrm{~h}$. The specific surface areas were evaluated with the Brunauer-Emmett-Teller (BET) method in the $P / P_{0}$ range of $0.05-0.3$. Pore size distribution curves were calculated from the adsorption branch of the isotherms with the Barrett-Joyner-Halenda $(\mathrm{BJH})$ method, and pore sizes were obtained from the peak positions of the distribution curves. The pore volume was taken at the $P / P_{0}=0.990$ point. 
The loading of the aminopropyl groups was calculated from the nitrogen content by elemental analysis, which was performed on a Heraeus CHNS elemental analyzer.

Thermogravimetric (TG) analyses were carried out on a Du Pont 951 thermogravimetric analyzer with a heating speed of $10^{\circ} \mathrm{C} / \mathrm{min}$ in an air flow of $50 \mathrm{ml} / \mathrm{min}$.

Fourier transform infrared (FTIR) spectra were taken on a Nicolet Magna-IR 550 spectrometer with a resolution of $2 \mathrm{~cm}^{-1}$ by the $\mathrm{KBr}$ method.

The NMR experiments were carried out at ${ }^{29} \mathrm{Si},{ }^{13} \mathrm{C}$, and ${ }^{1} \mathrm{H}$ frequencies of 59.6, 75.5, and $300.1 \mathrm{MHz}$, respectively, on a Bruker DSX300 NMR spectrometer equipped with a commercial 4-mm MAS-NMR probe. All spectra were measured at room temperature. The magic-angle spinning frequencies were set at $6 \mathrm{kHz}$ for all experiments, and the variation was limited to $\pm 3 \mathrm{~Hz}$ with a commercial pneumatic control unit. Chemical shifts were externally referenced to TMS for ${ }^{29} \mathrm{Si}$ and ${ }^{13} \mathrm{C}$. For the ${ }^{29} \mathrm{Si}$ Bloch-decay experiment, the number of scans was set at 3072 , and the recycle delay was set at $60 \mathrm{~s} .{ }^{13} \mathrm{C}\left\{{ }^{1} \mathrm{H}\right\}$ cross-polarization spectra were measured with a recycle delay of $5 \mathrm{~s}$, and the contact times were $3 \mathrm{~ms}$. The number of scans was 5120. During the contact time the ${ }^{1} \mathrm{H}$ nutation frequency was set equal to $50 \mathrm{kHz}$, and a linear ramping was applied to the nutation frequencies of ${ }^{13} \mathrm{C}$ (27.3 to $43.0 \mathrm{kHz}$ ). The proton decoupling field during the acquisition period was $83 \mathrm{kHz}$.

\subsection{Catalytic reaction procedure}

The catalytic reactions of flavanone synthesis were carried out under $\mathrm{N}_{2}$ in a sealed flask immersed in a thermostatted bath with a magnetic stirrer. For the reactions in the absence of solvent, $15 \mathrm{mmol}$ of benzaldehyde derivative and $10 \mathrm{mmol}$ of substituted $2^{\prime}$-hydroxyacetonphenone were mixed and heated to $140^{\circ} \mathrm{C}$, and then $0.15 \mathrm{~g}$ of the dried catalyst was rapidly added. For the reactions in the presence of solvent, benzaldehyde and 2 '-hydroxyacetophenone were mixed with $5 \mathrm{ml}$ of the solvent and heated to $140^{\circ} \mathrm{C}$ before the dried catalyst was added. The liquid products were separated from the reaction mixture at appropriate reaction intervals with a filtering syringe and diluted with chlorobenzene for analysis. The products were analyzed with a Chrompak CP 9000 gas chromatograph (GC) equipped with a $30 \mathrm{~m} \times$ $0.32 \mathrm{~mm}$ RTX-50 capillary column and FID detector, and identified by GC-mass spectrometry (HP5971 mass spectrometer connected to a $30 \mathrm{~m} \times 0.25 \mathrm{~mm}$ RTX-50 capillary column). To calculate the weight balance at the end of reaction, the catalyst was filtered and washed with $\mathrm{CH}_{2} \mathrm{Cl}_{2}$. Then the solid was refluxed in fresh $\mathrm{CH}_{2} \mathrm{Cl}_{2}$ for $3 \mathrm{~h}$, followed by filtration and washing again with $\mathrm{CH}_{2} \mathrm{Cl}_{2}$. The organic materials were recovered after the solvent was evaporated. It accounted for more than $95 \%$ of the starting reactants.
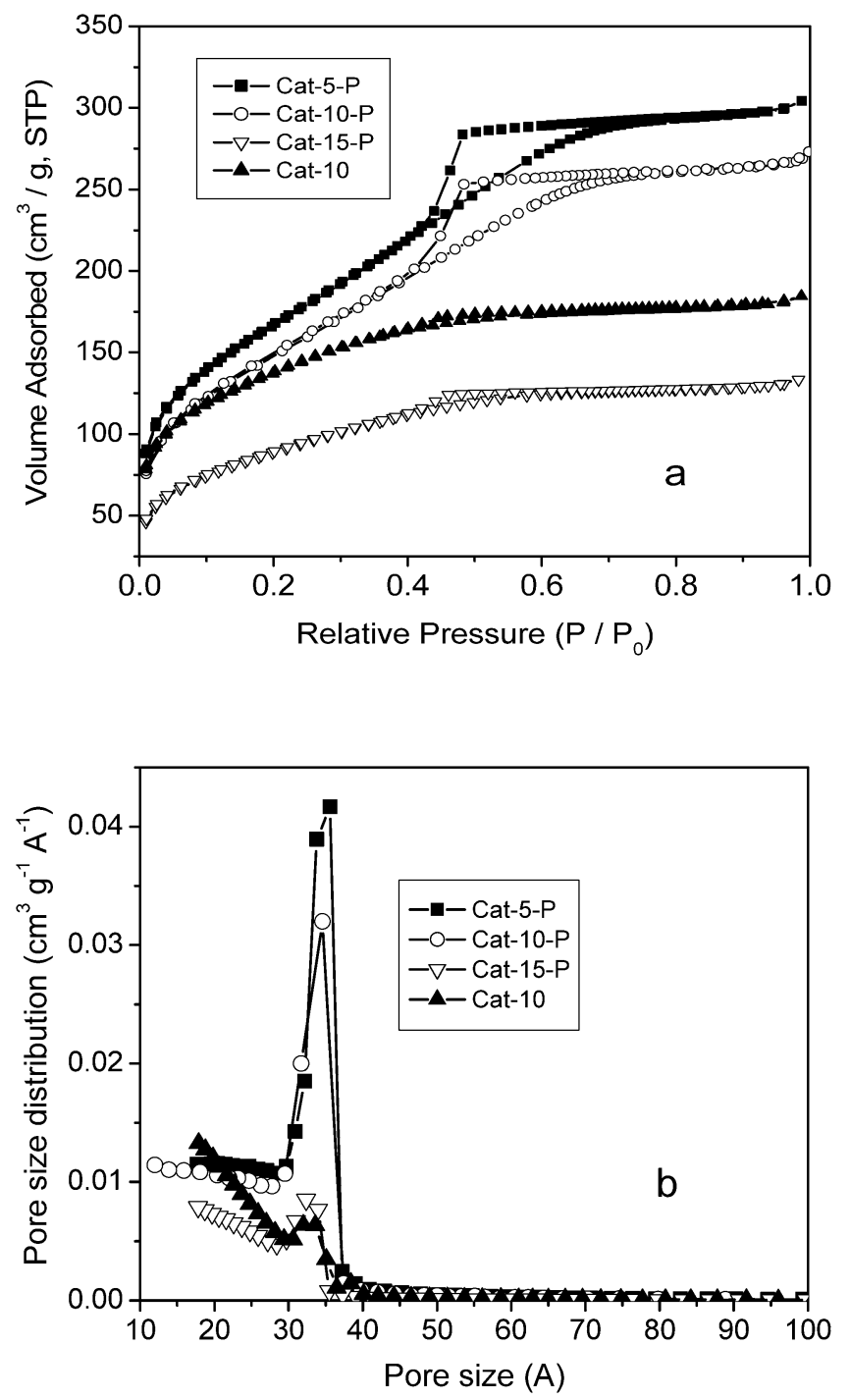

Fig. 1. (a) Nitrogen adsorption-desorption isotherms and (b) BJH pore size distribution of the aminopropylated silica materials.

\section{Results and discussion}

\subsection{Catalyst characterization}

\subsection{1. $N_{2}$ sorption and elemental analysis}

The $\mathrm{N}_{2}$ adsorption-desorption isotherms of the catalysts are shown in Fig. 1. The isotherms of Cat-5-P and Cat-10-P have type IV characteristics of the IUPAC classification, and those of Cat-15-P and Cat-10 have no apparent hysteresis loops and are closer to type I characteristics. BJH pore size analysis shows that Cat-5-P and Cat-10-P contain mesopores of narrow pore size distribution (PSD) in the range of 3-4 nm, and Cat-15-P and Cat-10 contain only negligible amounts of mesopores. These results imply that mesoporous structures of narrow PSD can be formed by prehydrolysis of TEOS and then co-condensation with an appropriate amount of APTES in an acidic environment without the addition of pore-directing agents. Nevertheless, these materials were 
Table 1

Physicochemical properties of the aminopropylated amorphous silica and SBA- $\mathrm{NH}_{2}$ catalysts

\begin{tabular}{lllll}
\hline Catalyst & $\begin{array}{l}S_{\mathrm{BET}} \\
\left(\mathrm{m}^{2} / \mathrm{g}\right)\end{array}$ & $\begin{array}{l}V_{\mathrm{p}} \\
\left(\mathrm{cm}^{3} / \mathrm{g}\right)\end{array}$ & \multicolumn{2}{c}{ N content $(\mathrm{mmol} / \mathrm{g})$} \\
\hline Cat-0 & 753 & 0.62 & 0 & 0 \\
Cat-5-P & 609 & 0.46 & 0.8 & 0.67 \\
Cat-10-P & 542 & 0.39 & 1.54 & 1.62 \\
Cat-15-P & 310 & 0.20 & 2.22 & 2.18 \\
Cat-10 & 489 & 0.28 & 1.54 & 1.57 \\
SBA-NH & 664 & 0.85 & 1.54 & 1.21 \\
\hline
\end{tabular}

X-ray amorphous, implying no long-range ordering of the mesopores.

The basic physical and compositional characteristics are shown in Table 1. The aminopropyl groups from APTES are effectively incorporated into the silica framework. However, the surface area and pore volume decrease with nitrogen content for the materials prepared by the TEOS prehydrolysis process. On the other hand, in a comparison of Cat-10-P and Cat-10, which have the same APTES content in the mixture but are prepared by different methods, the one prepared by TEOS prehydrolysis has a larger surface area, pore volume, and narrower PSD than that prepared without prehydrolysis.

\subsubsection{Thermogravimetric analysis}

The weight loss (TGA) and derivative weight (DTA) of the prepared catalysts containing $10 \mathrm{~mol} \%$ APTES in the initial mixture with and without TEOS prehydrolysis are illustrated in Fig. 2. The relatively large weight losses at temperatures lower than $100^{\circ} \mathrm{C}$ are attributed to the loss of adsorbed water. However, the amount of adsorbed water decreased from $17 \mathrm{wt} \%$ for Cat-10-P synthesized with TEOS prehydrolysis to $12 \mathrm{wt} \%$ for Cat-10 without prehydrolysis, indicating the greater surface hydrophilicity of the former catalyst [25]. This may be due to the fact that the amino groups on Cat-10-P, which contain mesopores of narrow PSD, are more accessible by water than are those on Cat- 10 . The weight loss of about $8-10 \mathrm{wt} \%$ from 250 to $500^{\circ} \mathrm{C}$ is due to the decomposition of the aminopropyl groups incorporated. Furthermore, both samples exhibit about $1 \mathrm{wt} \%$ weight loss at $500-700^{\circ} \mathrm{C}$. These are likely due to the dehydroxylation of the silicate networks [26] or the elimination of residual ethoxy groups, attributed to the incomplete hydrolysis of TEOS [27]. The results of TG analysis were in agreement with those of the chemical elemental analyses, revealing that APTES in the synthesis mixture was almost completely incorporated into the silica framework.

\subsubsection{FTIR measurement}

Fig. 3 illustrates the FTIR spectra of the aminopropylated silicas with different APTES contents prepared with and without TEOS prehydrolysis. It shows that TEOS prehydrolysis in the preparation procedure does not exert an obvious influence on the IR spectra. The typical $\mathrm{Si}-\mathrm{O}-\mathrm{Si}$ bands
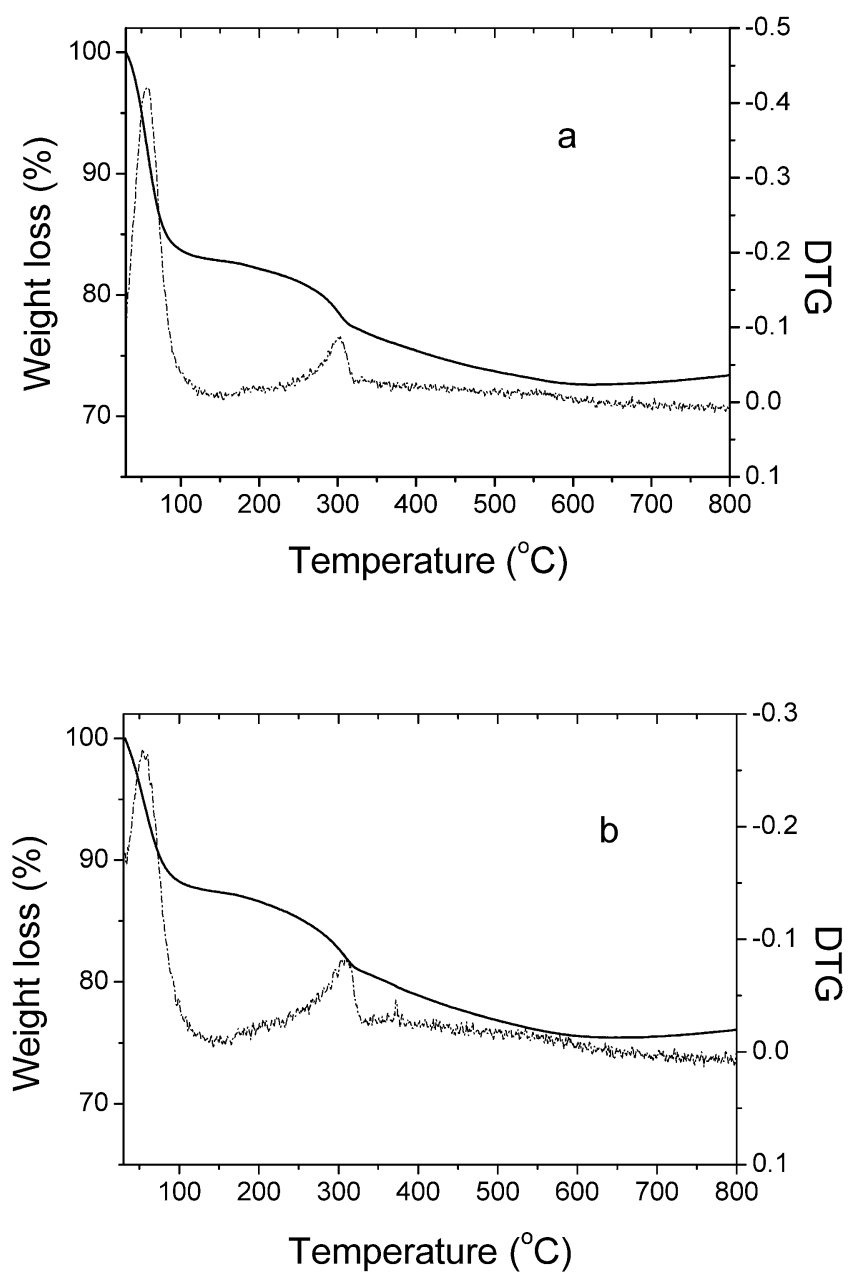

Fig. 2. TGA and DTG profiles of aminopropylated silica containing $10 \mathrm{~mol} \%$ APTES (a) with and (b) without TEOS prehydrolysis.

around 1220, 1070, 791, and $470 \mathrm{~cm}^{-1}$ associated with the condensed silica network are present in all cases. In the lowfrequency region, the presence of the weak $\mathrm{N}-\mathrm{H}$ bending vibration at $687 \mathrm{~cm}^{-1}$ and the symmetric $-\mathrm{NH}_{3}{ }^{+}$bending vibration around $1510 \mathrm{~cm}^{-1}$ confirm the incorporation of amino groups. The intensity of these two peaks increases with the APTES content. The peak of the $\mathrm{C}-\mathrm{N}$ stretching vibration is normally observed in the range of 1000$1200 \mathrm{~cm}^{-1}$ [28]. However, this peak cannot be resolved, because of its overlap with the absorbance of $\mathrm{Si}-\mathrm{O}-\mathrm{Si}$ stretch in the $1000-1130 \mathrm{~cm}^{-1}$ range and that of $\mathrm{Si}-\mathrm{CH}_{2}-\mathrm{R}$ stretch in the $1200-1250 \mathrm{~cm}^{-1}$ range $[27,29]$. The weak bands around $1450-1470 \mathrm{~cm}^{-1}$, associated with $-\mathrm{CH}_{2}$ vibrations, can be seen for the samples containing aminopropyl groups and the intensity increases with the APTES content (Fig. 3B). This further confirms the incorporation of organic species into the framework. For the reference silica sample prepared by the same method, no absorbance peaks at 1475,1450 , or $1385 \mathrm{~cm}^{-1}$, ascribed to $\delta_{\text {asym }}-\mathrm{CH}_{3}$, $\delta-\mathrm{CH}_{2}$, and $\delta_{\text {sym }}-\mathrm{CH}_{3}$, respectively, can be seen, implying that ethoxy groups in TEOS were completely hydrolyzed under the synthesis conditions [23]. The pres- 

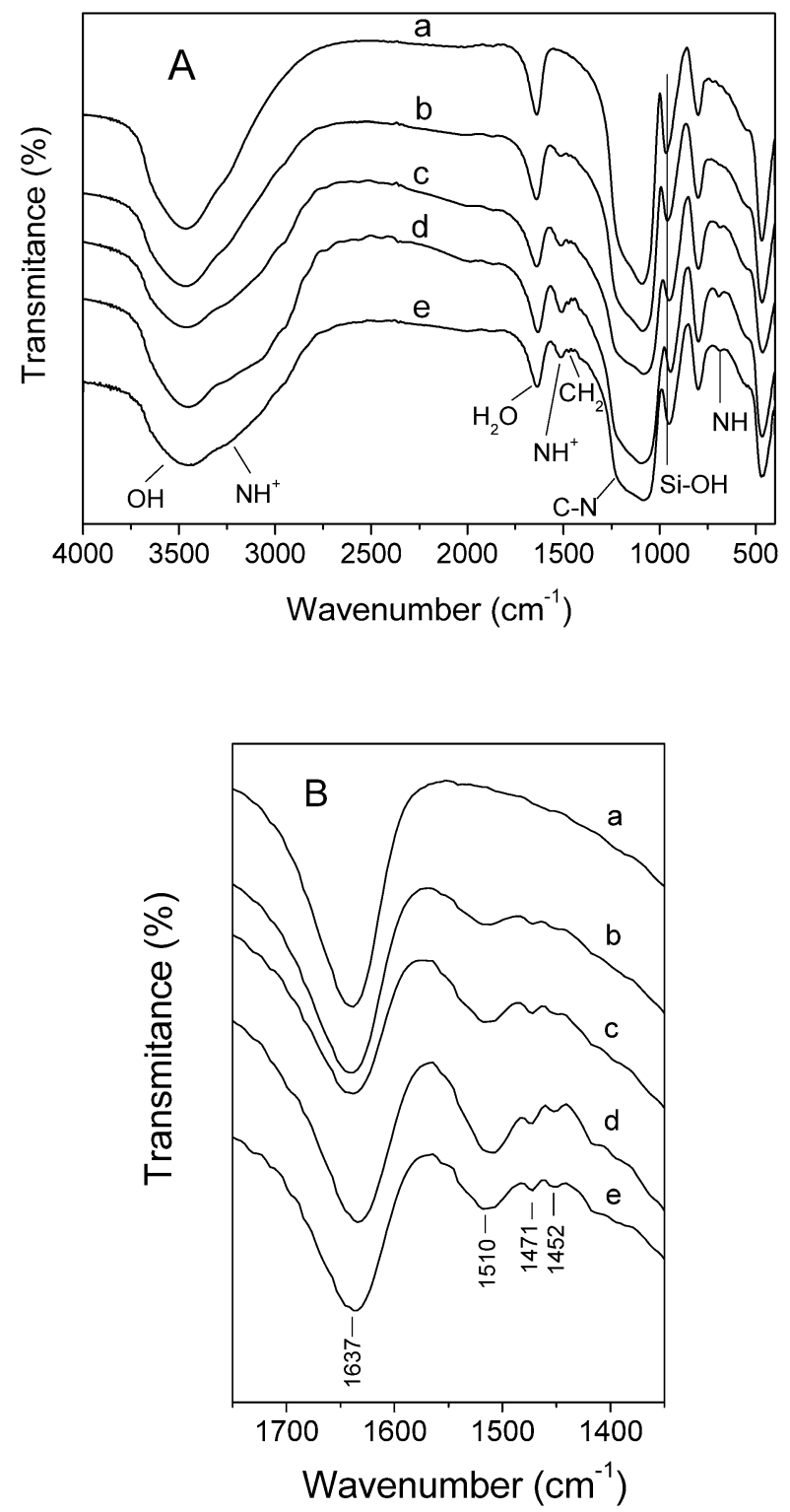

Fig. 3. FTIR spectra of samples prepared with TEOS prehydrolysis containing different APTES contents: (a) $0 \mathrm{~mol} \%$, (b) $5 \mathrm{~mol} \%$, (c) $10 \mathrm{~mol} \%$, (d) $15 \mathrm{~mol} \%$, and (e) a sample prepared without TEOS prehydrolysis containing $10 \mathrm{~mol} \%$ APTES in the starting mixture (whole range in (A) and amplification between 1350 and $1750 \mathrm{~cm}^{-1}$ in (B)).

ence of two weak bands at 1990 and $1875 \mathrm{~cm}^{-1}$ is attributed to the overtones and combination modes of the inter- and intra-tetrahedral fundamental vibrational modes of the siliceous framework, which completely absorbs IR radiation below $1300 \mathrm{~cm}^{-1}$ [23]. The strong peak around $1630 \mathrm{~cm}^{-1}$ is mainly from the bending vibration of adsorbed $\mathrm{H}_{2} \mathrm{O}$. For all of the samples, the peaks associated with noncondensed $\mathrm{Si}-\mathrm{OH}$ groups in the range of 940 $970 \mathrm{~cm}^{-1}$ were present [30]. The gradual shift of the $\mathrm{Si}-\mathrm{OH}$ band toward lower wave number (from 966 to $942 \mathrm{~cm}^{-1}$ ) as the APTES concentration increases is probably due to the increase in interaction between the $-\mathrm{NH}_{2}$ groups and the silanol groups through hydrogen bonding, as illustrated

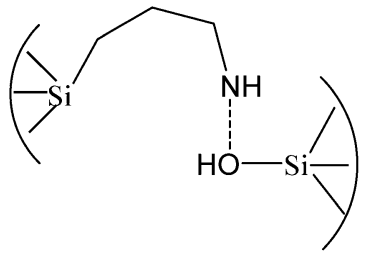

a

Fig. 4. Possible hydrogen bonding interaction (a) intermolecular and (b) intramolecular $\mathrm{Si}-\mathrm{OH} \cdots \mathrm{NH}_{2}$ bridges [23].

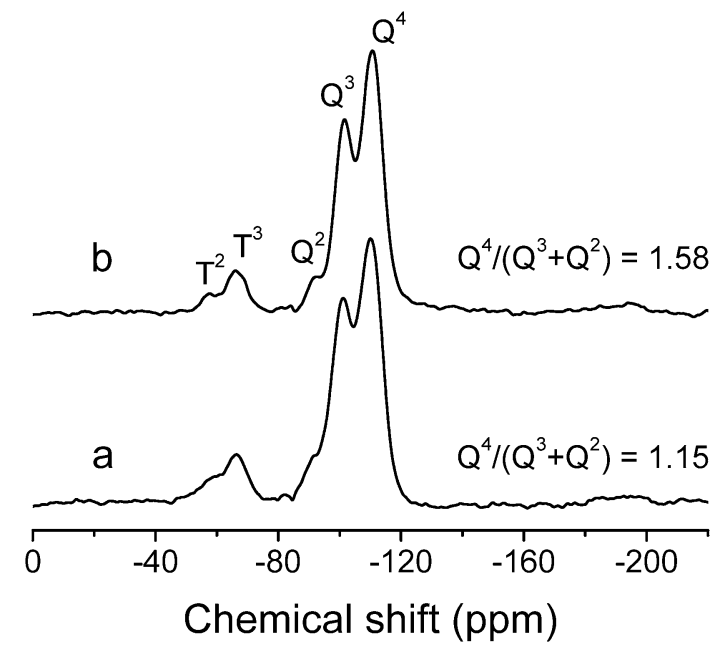

Fig. 5. ${ }^{29}$ Si MAS NMR spectra of aminopropylated silica materials containing $10 \mathrm{~mol} \%$ APTES in the mixture (a) with and (b) without TEOS prehydrolysis.

in Figs. $4 a$ and $b$ [23]. In the higher frequency region, the presence of aminopropyl groups was further corroborated by a broad band at 2700 to $3400 \mathrm{~cm}^{-1}$ and by the increases in its intensity with the aminopropyl content. The peaks in the $3000-2750 \mathrm{~cm}^{-1}$ range are due to the stretching of $\mathrm{CH}_{2}$ groups, and those in the $3000-3400 \mathrm{~cm}^{-1}$ range are attributed to the asymmetric and symmetric stretching vibrations of $\mathrm{NH}_{2}$ groups. In comparison with pure silica, the broadening of the silanol bands around $3480 \mathrm{~cm}^{-1}$ may be caused by the hydrogen-bonding interaction between the $-\mathrm{NH}_{2}$ groups and the silanol groups.

\subsubsection{Solid-state NMR}

Solid-state ${ }^{13} \mathrm{C}$ and ${ }^{29} \mathrm{Si}$ NMR spectroscopies were proved to be the most useful for providing chemical information regarding the condensation of organosiloxane and siloxane. ${ }^{29} \mathrm{Si}$ MAS NMR spectra for aminopropylated silica materials containing $10 \mathrm{~mol} \%$ APTES in the mixture are shown in Fig. 5. Three distinct resonance peaks at $-92,-101$, and $-110 \mathrm{ppm}$ are attributed to the $\mathrm{Q}^{2}, \mathrm{Q}^{3}$, and $\mathrm{Q}^{4}$ silicons $\left(\mathrm{Q}^{n}=\mathrm{Si}(\mathrm{OSi})_{n}(\mathrm{OH})_{4-n}, n=2-4\right)$, respectively, and two other peaks at -57 and $-67 \mathrm{ppm}$ are attributed to the $\mathrm{T}^{2}$ and $\mathrm{T}^{3}$ silicons $\left(\mathrm{T}^{m}=\mathrm{RSi}(\mathrm{OSi})_{m}(\mathrm{OH})_{3-m}, m=1-3\right)$, respectively. The appearance of $\mathrm{T}^{m}$ peaks confirms that the organosiloxane precursor APTES are condensed as a part of the silica framework. The relative integrated intensities of 


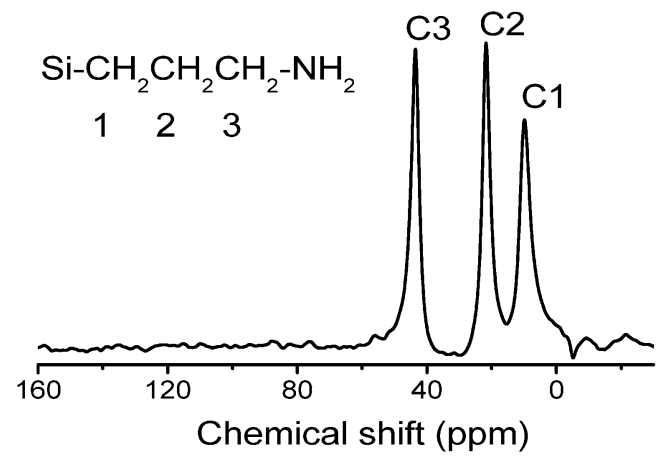

Fig. $6 .{ }^{13} \mathrm{C}$ CP MAS NMR spectra of aminopropylated silica materials containing $10 \mathrm{~mol} \%$ APTES in the mixture with TEOS prehydrolysis.

the $\mathrm{T}^{m}$ and $\mathrm{Q}^{n}$ signals $\left(\mathrm{T}^{m} /\left(\mathrm{T}^{m}+\mathrm{Q}^{n}\right)\right)$ are 0.12 and 0.11 for Cat-10-P and Cat-10, respectively, in good agreement with those theoretically expected on the basis of the composition of APTES in the initial mixture. The relative area ratios of $\mathrm{Q}^{4} /\left(\mathrm{Q}^{3}+\mathrm{Q}^{2}\right)$ peaks (Fig. 5) of these two samples are different, indicating that the preparation method has influence on the completeness of TEOS condensation. The $\mathrm{Q}^{4} /\left(\mathrm{Q}^{3}+\mathrm{Q}^{2}\right)$ ratio of 1.15 for the sample synthesized with TEOS prehydrolysis (Cat-10-P) is smaller than the value of 1.58 for Cat-10. Although Cat-10-P, with larger surface area and pore volume, may contain more silanol groups on the surface, the relatively smaller $Q^{4} /\left(Q^{3}+Q^{2}\right)$ value of Cat$10-\mathrm{P}$ in comparison with that of Cat-10 implies that larger numbers of defects may be present in the former sample. Since the catalysts were prepared in open beakers, when APTES was added in the synthesis gel of Cat-10-P after TEOS prehydrolysis, a portion of the water was evaporated. Therefore, the APTES concentration in the gel of Cat-10-P should be relatively high in comparison with that in the synthesis mixture of Cat-10. As a result, the dehydration and condensation of the silicate species were more seriously interfered with during the preparation of sample Cat-10-P than they were during the preparation of Cat-10.

The ${ }^{13} \mathrm{C}$ CP-MAS NMR spectrum of Cat-10-P is illustrated in Fig. 6. Three peaks at 10, 22, and $44 \mathrm{ppm}$, corresponding to the $\mathrm{C}$ atoms on the $\mathrm{Si}-\mathrm{CH}_{2}-\mathrm{CH}_{2}-\mathrm{CH}_{2}-\mathrm{NH}_{2}$ group, are clearly displayed in sequence from left to right [31]. It further confirms that APTES precursors are co-condensed into the prepared material, and the organic moieties are not decomposed during the preparation procedure. Furthermore, no peak signals around 19 and $60 \mathrm{ppm}$ assigned to the car- bons of surface ethoxy groups $\left(\mathrm{Si}-\mathrm{OCH}_{2} \mathrm{CH}_{3}\right)$ are seen, further implying that the ethoxy groups of both TEOS and APTES precursors are completely hydrolyzed in the preparation procedure [32]. This is also supported by IR spectra in Fig. 3.

\subsection{Catalysis}

\subsubsection{Catalytic activity in the presence of solvent}

The Claisen-Schmidt condensation of benzaldehyde and 2 -hydroxyacetophenone was reported to be catalyzed by acids or bases; the reaction paths are shown in Scheme 1. Pure silica gel Cat- 0 treated with a $0.2 \mathrm{M}$ methanol solution of TMAOH was first tested for the catalytic condensation between benzaldehyde (1a) and 2'-hydroxyacetophenone (2a), with and without the solvents. The reaction results showed that pure silica gave negligible conversion $(<3 \%)$ of acetophenone in all cases. In contrast, significant conversions and selectivities of flavanone (4a) were obtained over the aminopropylated silica. Table 2 shows that the catalyst gave different conversions of $2^{\prime}$-hydroxyacetophenone and selectivities of $2^{\prime}$-hydroxychalcone (3a) and flavanone in different solvents and under solvent-free conditions over Cat10-P. However, there seems to be no correlation between the solvent polarity and the conversion or the selectivity for flavanone. Entry 6 in Table 2 shows that the catalyst under solvent-free conditions has much a higher conversion of 2a and selectivity for flavanone, indicating that solvent can hamper both the conversion of the reactants and the isomerization of chalcone. This may be due to dilution of the concentration of the reactant by the solvent molecules, which would reduce the contact probability between the reactant molecules and the base active sites on the catalyst. On the other hand, the solvent molecules surrounding the amine active sites also reduce their ability to interact with the reactants. The decrease in the selectivity for flavanone can be responsible for the change in thermodynamic balance between chalcone and flavanone in different solvents. These results are different from those obtained over other solid catalysts such as $\mathrm{MgO}$ [12] and natural phosphate [33], in which the solvent often facilitates the conversion of the reactants and the isomerization of chalcone. Moreover, no byproducts other than chalcone and flavanone were observed in our reaction products, indicating that Cannizzaro reaction of

Table 2

The catalytic performance of Cal-10-P in the condensation of benzaldehyde and $2^{\prime}$-hydroxyacetophenone with and without solvents at $140{ }^{\circ} \mathrm{C}$ for $10 \mathrm{~h}$

\begin{tabular}{llllll}
\hline Entry & Solvent & $\begin{array}{l}\text { Dielectric constant } \\
\left(20^{\circ} \mathrm{C}\right)\end{array}$ & $\begin{array}{l}\text { Conversion of } \\
\mathbf{2 a}(\%)\end{array}$ & $\begin{array}{l}\text { Selectivity to 3a } \\
(\%)\end{array}$ \\
\hline 1 & DMSO & 48.9 & 30 & 36 & $\begin{array}{l}\text { Selectivity to 4a } \\
(\%)\end{array}$ \\
2 & Nitrobenzene & 35.6 & 35 & 45 & 64 \\
3 & Benzonitrile & 26.5 & 13 & 40 & 55 \\
4 & 1,2-Dichlorobenzene & 2.8 & 17 & 52 & 40 \\
5 & $1,3,5$-Trimethylbenzene & 2.3 & 20 & 44 & 56 \\
6 & - & - & 88 & 31 & 69 \\
\hline
\end{tabular}




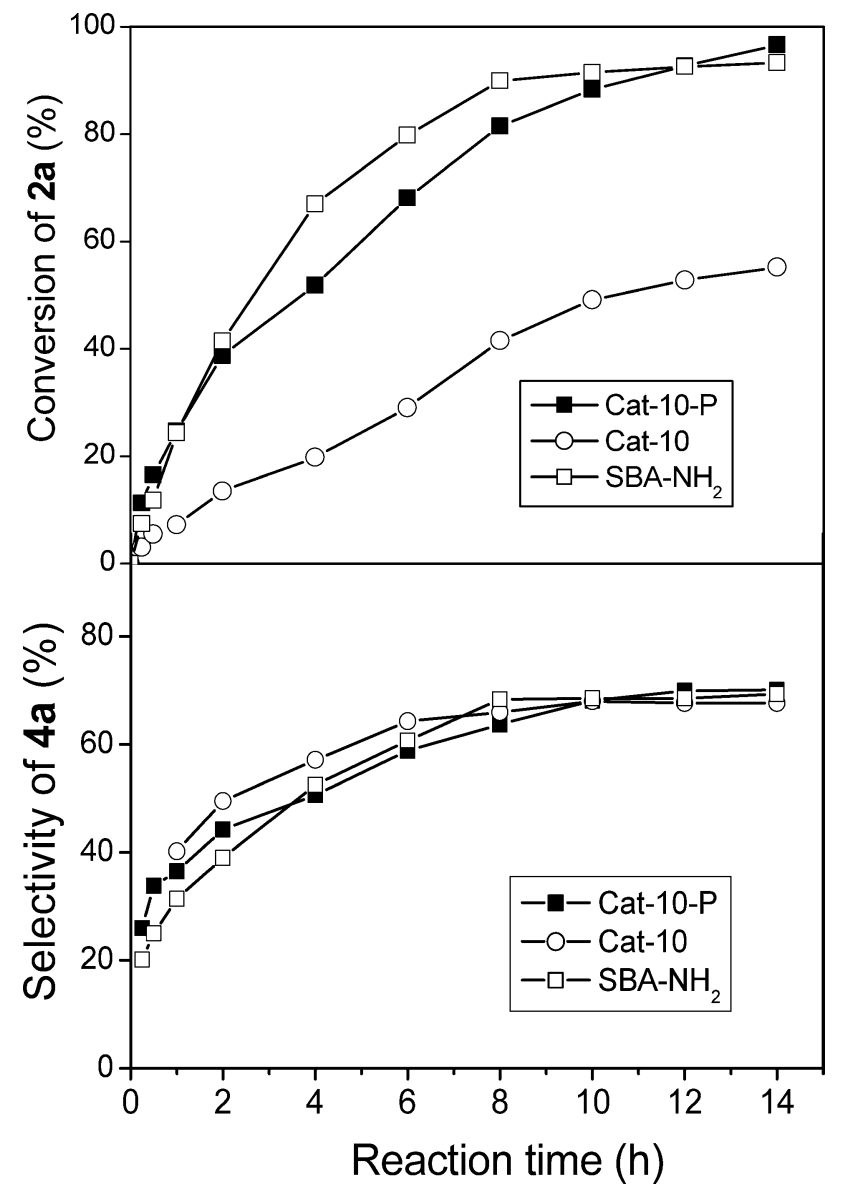

Fig. 7. The catalytic performances as a function of reaction time in the condensation of benzaldehyde and $2^{\prime}$-hydroxyacetophenone in the absence of solvent over aminopropylated silica materials prepared by different methods.

aldehyde or condensation of ketone did not take place under the reaction conditions.

\subsubsection{Dependence of reaction period}

The catalytic activities versus the reaction time in Claisen-Schmidt condensation of benzaldehyde and 2'-hydroxyacetophenone over the aminopropylated silica materials prepared with $10 \mathrm{~mol} \%$ APTES but by different methods are shown in Fig. 7, including the amorphous silica synthesized with and without TEOS prehydrolysis (samples Cat-10-P and Cat-10, respectively), as well as SBA- $\mathrm{NH}_{2}$. The activities of Cat-10-P and SBA-NH $\mathrm{NH}_{2}$ were found to be close to each other, and both were almost twice as high as that of Cat10. As the reaction time increased to $14 \mathrm{~h}$, the conversion of 2a over Cat-10-P and SBA- $\mathrm{NH}_{2}$ increased to ca. 97\%, whereas that over Cat-10 was only $55 \%$. Since both Cat-10$\mathrm{P}$ and SBA- $\mathrm{NH}_{2}$ possess mesopores of narrow PSD, these results indicate that the presence of mesopores has a great influence on the performance of the catalysts, which likely facilitates the diffusion of the reactant molecules to the active sites and the product molecules away from the surface of the catalyst. This proposal was also confirmed by the observation that the reaction reaches the highest conversion faster
Table 3

Catalytic performance of various aminopropylated catalysts with different amino loadings and preparation methods in the condensation of benzaldehyde and $2^{\prime}$-hydroxyacetophenone in the absence of solvent at $140{ }^{\circ} \mathrm{C}$ for $10 \mathrm{~h}$

\begin{tabular}{llll}
\hline Catalyst & $\begin{array}{l}\text { Conversion of 2a } \\
(\%)\end{array}$ & $\begin{array}{l}\text { Selectivity to 3a } \\
(\%)\end{array}$ & $\begin{array}{l}\text { Selectivity to 4a } \\
(\%)\end{array}$ \\
\hline Cat-5-P & 76 & 31 & 69 \\
Cat-10-P & 88 & 31 & 69 \\
Cat-15-P & 24 & 31 & 69 \\
Cat-10 & 49 & 32 & 68 \\
Used Cat-10-P & 64 & 30 & 70 \\
\hline
\end{tabular}

over SBA-NH $\mathrm{N}_{2}$, which possesses highly ordered mesopores, rather than over Cat-10-P. Nevertheless, the latter catalyst was obtained by a much more economical route without the use of an organic template. On the other hand, the selectivities for flavanone over all three catalysts were similar. The selectivity for flavanone increased gradually within $8-10 \mathrm{~h}$, and then it retained around $69 \%$ as the reaction time was prolonged to $14 \mathrm{~h}$. This is due to the thermodynamic equilibrium between 2 '-hydroxychalcone and flavanone, which was supported by a separate experiment. When $0.15 \mathrm{~g}$ of the catalyst was heated with flavanone at $140^{\circ} \mathrm{C}$ for more than $8 \mathrm{~h}$, a portion of the flavanone was converted to chalcone, and the molar ratio of flavanone/(flavanone + chalcone) was around $69 \%$, and that was unchanged with prolonged reaction time.

\subsubsection{Influence of aminopropyl group loading}

The catalytic activities of the catalysts containing different amounts of amino groups are displayed in Table 3. Both Cat-5-P and Cat-10-P catalysts, which contain mesoporous structures with narrow PSDs, show very high conversions of 2a (76 and $88 \%$, respectively). In contrast, Cat-15-P, which contains greater amounts of amino groups but a negligible amount of mesopores, has the conversion dramatically decreased to $24 \%$. However, the selectivities for flavanone are all similar and are around 69\%.

When the used Cat-10-P catalyst was re-used after being treated with $\mathrm{CH}_{2} \mathrm{Cl}_{2}$ in a Soxhlet apparatus for $3 \mathrm{~h}$ and then dried at $100{ }^{\circ} \mathrm{C}$ overnight, a lower conversion of acetophenone (64 versus $88 \%$ ) but a similar selectivity for flavanone (70 versus 69\%) was observed (Table 3). These results indicate that most of the active sites could be regenerated by solvent treatment, and the decrease in conversion is probably due to the incomplete removal of absorbed species from the active sites by a simple $\mathrm{CH}_{2} \mathrm{Cl}_{2}$ treatment.

\subsubsection{Influence of the nature of the reactant}

The results of Claisen-Schmidt condensations between various para-substituted benzaldehyde and $2^{\prime}$-hydroxyl- $5^{\prime}$ substituted acetophenones carried out in the absence of solvent over Cat-10-P are summarized in Table 4. All of the reactions show relatively high conversions of acetophenones and very high selectivities for flavanones. To the best of our knowledge, the selectivities for flavanones reported in this table are the highest values reported for the catalytic 
Table 4

Reactions of benzaldehyde and $2^{\prime}$-hydroxyacetophenone with various substituting groups in the absence of solvent over Cat-10-P at $140^{\circ} \mathrm{C}$ for $10 \mathrm{~h}$

\begin{tabular}{llllll}
\hline Reaction & $\mathrm{R}$ & $\mathrm{R}^{\prime}$ & $\begin{array}{l}\text { Conversion } \\
\text { of } 2(\%)\end{array}$ & $\begin{array}{l}\text { Selectivity } \\
\text { to } \mathbf{3}(\%)\end{array}$ & $\begin{array}{l}\text { Selectivity } \\
\text { to } \mathbf{4}(\%)\end{array}$ \\
\hline $\mathrm{a}$ & $\mathrm{H}$ & $\mathrm{H}$ & 88 & 31 & 69 \\
$\mathrm{~b}$ & $\mathrm{CH}_{3} \mathrm{O}$ & $\mathrm{H}$ & 94 & 45 & 55 \\
$\mathrm{c}$ & $\mathrm{Cl}$ & $\mathrm{H}$ & 86 & 30 & 70 \\
$\mathrm{~d}$ & $\mathrm{NO}_{2}$ & $\mathrm{H}$ & 58 & 15 & 85 \\
$\mathrm{e}$ & $\mathrm{H}$ & $\mathrm{CH}_{3} \mathrm{O}$ & 83 & 14 & 86 \\
$\mathrm{f}$ & $\mathrm{H}$ & $\mathrm{Cl}$ & 90 & 24 & 76 \\
$\mathrm{~g}$ & $\mathrm{CH}_{3} \mathrm{O}$ & $\mathrm{CH}_{3} \mathrm{O}$ & 80 & 18 & 82 \\
$\mathrm{~h}$ & $\mathrm{CH}_{3} \mathrm{O}$ & $\mathrm{Cl}$ & 90 & 32 & 68 \\
$\mathrm{i}$ & $\mathrm{Cl}$ & $\mathrm{CH}_{3} \mathrm{O}$ & 55 & 13 & 87 \\
$\mathrm{j}$ & $\mathrm{NO}_{2}$ & $\mathrm{CH}_{3} \mathrm{O}$ & 60 & 21 & 79 \\
\hline
\end{tabular}

Claisen-Schmidt condensation reactions with or without the solvents up to date.

The substituting groups in the aromatic rings have a great influence on the conversion and selectivity. Reactions a-d show that the presence of electron-donating groups at the para position of benzaldehyde (1) increases the conversion of the reactants but decreases the selectivity for flavanones, and the electron-withdrawing groups on benzaldehyde decrease the conversion but increase the flavanone selectivity. These trends in conversion are the reverse of those observed on other solid base or acid catalysts [16,20], implying that the Claisen-Schmidt condensation may have a different reaction mechanism over the aminopropylated silica. In contrast, the electron-donating groups in the aromatic rings of acetophenone (2) decrease the conversion of the reactants, and the electron-withdrawing groups increase the conversion of the reactants (reactions e and f). However, the influence of the substituents on acetophenone was less significant in comparison with that on benzaldehyde. Moreover, both the electron-donating and electron-withdrawing groups on acetophenone enhance the selectivity for flavanones. The steric effect of substituting groups such as $\mathrm{NO}_{2-}, \mathrm{Cl}-$, and $\mathrm{CH}_{3} \mathrm{O}$ - was reported to decrease catalytic activities over other solid catalysts like barium hydroxide, zeolites, and hydrotalcites [14-18]. However, this seems to be negligible in our system, which may be due to the relatively large space in the mesopore structure.

\subsubsection{Reaction mechanism}

The Claisen-Schmidt condensation of benzaldehyde and $2^{\prime}$-hydroxyacetophenone has been studied under acidic or basic conditions [7-9]. When a base catalyst is used, the reaction mechanism generally accepted involves the formation of the anion of acetophenone through the deprotonation of the methyl group, followed by its attack on the carbonyl group on benzaldehyde. If the reaction condition and the substituents present in the aromatic rings are suitable, the chalcone products can cyclize to form the corresponding flavanone isomers [7-9,16]. According to these pathways, the aldehydes bearing the electron-withdrawing groups in the aromatic ring should be more reactive than those with electron-donating groups. However, the opposite was observed in our system, with aminopropylated silica as the catalyst. This suggests that the reaction mechanism could be different from that over other base catalysts. The previous studies on the adsorption of benzaldehyde on aminopropyl xerogels by Sartori et al. $[23,34]$ showed that the $\mathrm{C}=\mathrm{N}$ imine species were formed as the main component, and on the basis of imine formation a reaction mechanism for the nitroaldol condensation was proposed. To explain the present reaction results, the imine species were proposed to be one of the intermediates. The modified reaction mechanism is shown in Scheme 2. Over the aminopropylated silica catalysts, the benzaldehyde molecules and the amino groups would form the $\mathrm{C}=\mathrm{N}$ imine species, which was then attacked by the anion of acetophenone to produce the corresponding adduct. According to the organic textbook [35], the first step of the imine formation is the attack by the nucleophilic amine on the carbonyl. A rapid proton transfer results in an unstable intermediate called a carbinolamine. A carbinolamine then reacts to form imine by the loss of water. In the homogeneous system of imine formation, it was reported that under slightly acidic conditions, formation of the carbinolamine intermediate is slow, while under neutral or basic conditions, dehydration of the carbinolamine becomes the slow step [36]. An electron-withdrawing group on benzaldehyde would favor the formation of carbinolamine intermediate but hinder the dehydration process and the formation of imine. As a result, the conversion decreases. According to the reaction results, imine formation should be the key step in determining the conversion. Furthermore, the electron-withdrawing group on benzaldehyde facilitates the subsequent chalcone isomerization, and the selectivity of flavanone increases. As for the substituents at the meta position of acetophenone, the influence on the conversion was found to be less significant. However, the selectivities for flavanone were increased with substituents on the acetophenone. This is probably the thermodynamic distribution between products 3 and 4 .

\section{Conclusions}

The aminopropylated silica catalysts with different loadings of the amino group were prepared by a sample cocondensation of TEOS and APTES under strong acid conditions. The materials with TEOS prehydrolysis and $10 \%$ or less APTES in the initial mixture contained mesoporous structure with narrow pore size distribution. They also showed larger surface area and pore volume than that without TEOS prehydrolysis. When used as base catalysts in liquid-phase reaction, the aminopropylated silica gave very high conversion and selectivity for flavanones for the Claisen-Schmidt condensation between substituted benzaldehydes and substituted $2^{\prime}$-hydroxyacetopheones and the subsequent isomerization of the $2^{\prime}$-hydroxychalcone intermediates under solvent-free conditions. Moreover, the ma- 


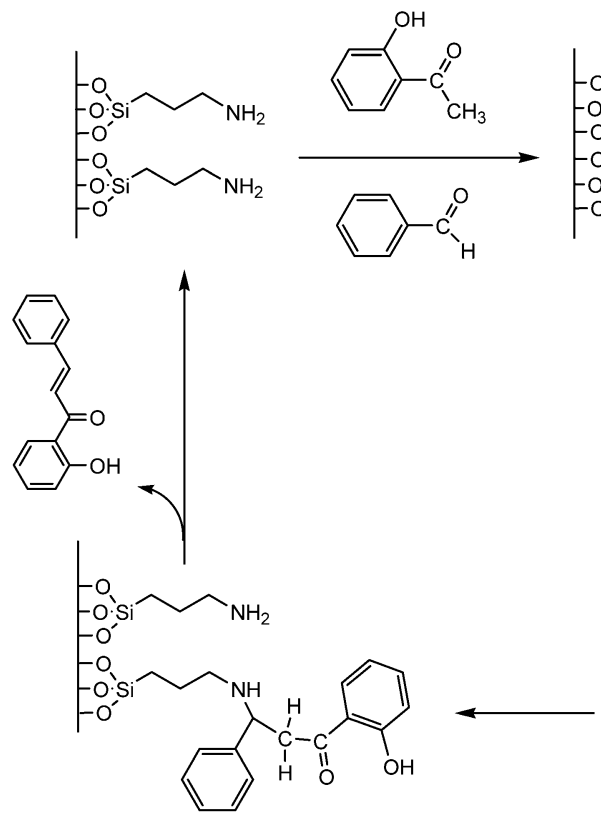

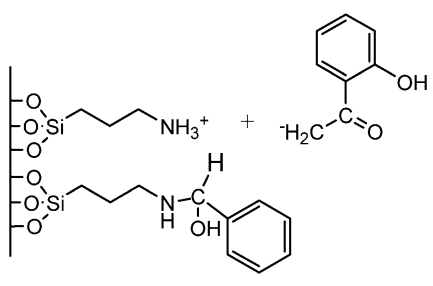

(i)
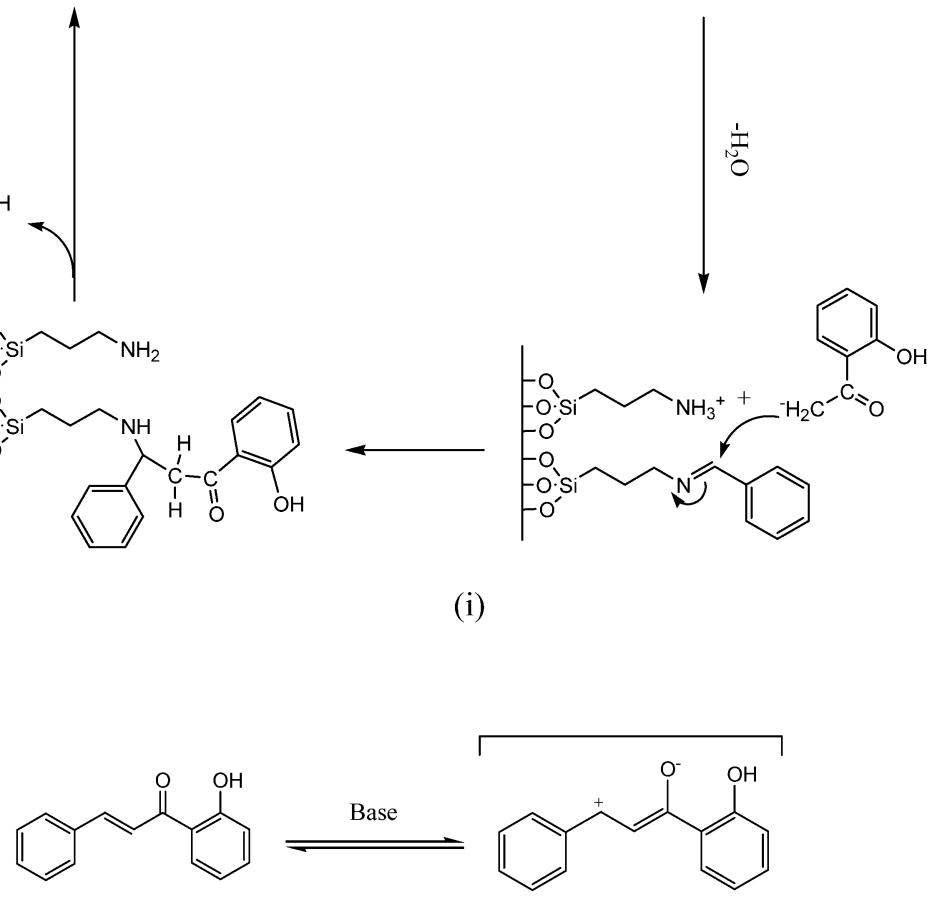

3

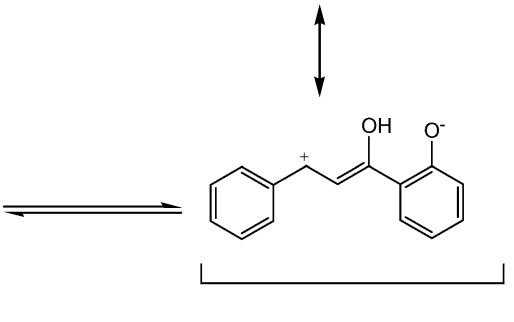<smiles>O=C1CC(c2ccccc2)Oc2ccccc21</smiles>

4

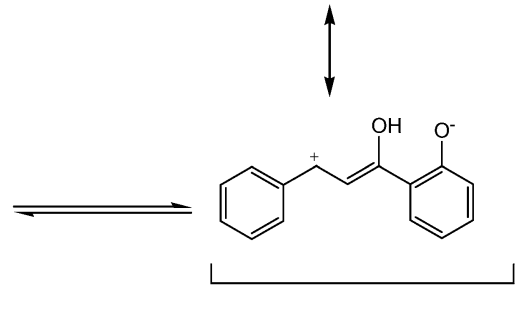

(ii)

Scheme 2. Proposed mechanisms over the aminopropylated silica for (i) Claisen-Schmidt condensation and (ii) isomerization of $2^{\prime}$-hydroxychalcone.

terials with narrower distributed mesopores showed much higher conversions. In contrast, the use of solvents markedly decreased both the catalytic activity and the selectivity for flavanone over the aminopropylated silica catalysts. The substituents in the aromatic rings of the benzaldehyde greatly influenced the conversion and the selectivity for flavanones, whereas the substituents at the meta position of acetophenone affected the conversion less significantly. The presence of electron-donating groups at the para position of benzaldehyde increased the conversion but decreased the selectivity for flavanones, whereas the electron-withdrawing groups on benzaldehyde decreased the conversion but increased the flavanone selectivity. The trend was in contrast to the results observed over other base catalysts. The pos- sible reaction mechanism through the imine intermediate was proposed. According to the reaction results, the imine formation should be the key step determining the conversion.

\section{Acknowledgments}

This project was supported by the National Science Council, Taiwan. S.C. thanks Profs. Y.-M. Tsai, S.-T. Liu and S.-H. Chiu at Department of Chemistry, National Taiwan University, for the valuable discussions on the reaction mechanism. 


\section{References}

[1] R. Li, G.L. Kenyon, F.E. Cohen, X. Chen, B. Gong, J.N. Dominguez, E. Davison, G. Kurzban, R.E. Miller, E.O. Nuzman, J. Med. Chem. 38 (1995) 5031.

[2] A.T. Dinkova-Kostova, C. Abeygunawardana, P. Talaley, J. Med. Chem. 41 (1998) 5278.

[3] J.F. Ballesteros, M.J. Sanz, A. Ubeda, M.A. Miranda, S. Iborra, M. Paya, M.J. Alcaraz, J. Med. Chem. 38 (1995) 2794.

[4] J.R. Dimmock, N.M. Kandepu, M. Hetherington, J.W. Quail, U. Pugazhenthi, A.M. Sudom, M. Chamankhah, P. Rose, E. Pass, T.M. Alle, S. Halleran, J. Szydlowski, B. Mutus, M. Tannous, E.K. Manavathu, T.G. Myers, E.D. Clercq, J. Balzani, J. Med. Chem. 41 (1998) 1014.

[5] M.D. Ankhiwala, J. Indian Chem. Soc. 67 (1990) 913.

[6] A. Mantas, E. Deretey, F.H. Ferretti, M.R. Estrada, I.G. Csizmadia, THEOCHEM 504 (2000) 171.

[7] D.N. Dahr, The Chemistry of Chalcones and Related Compounds, Wiley, New York, 1981.

[8] J.B. Harbone, T.J. Mabry, H. Mabry, The Flavonoids, Academic Press, New York, 1976.

[9] J.B. Harbone, T.J. Mabry, The Flavonoids: Advances in Research, Chapman \& Hall, New York, 1982.

[10] R.A. Sheldon, R.S. Downing, Appl. Catal. A Gen. 189 (1999) 163.

[11] M. Besson, M.C. Bonnet, P. Gallezot, I. Tkatchenko, A. Tuel, Catal. Today 51 (1999) 547.

[12] M.T. Drexler, M.D. Amiridis, J. Catal. 214 (2003) 136.

[13] R.S. Varma, G.W. Kabalka, L.T. Evans, R.M. Pagni, Synth. Commun. 15 (1985) 279.

[14] A. Aguilera, A.R. Alcantara, J.M. Marinas, J.V. Sinisterra, Can. J. Chem. 65 (1987) 1165.

[15] A. Fuentes, J.M. Marinas, J.V. Sinisterra, Tetrahedron Lett. 28 (1987) 4541.

[16] M.J. Climent, A. Corma, S. Iborra, J. Primo, J. Catal. 151 (1995) 60.
[17] A. Guida, M.H. Lhouty, D. Tichit, F. Figueras, P. Geneste, Appl. Catal. A 164 (1997) 251.

[18] M.J. Climent, A. Corma, S. Iborra, A. Velty, J. Catal. 221 (2004) 474.

[19] S. Sebti, A. Solhy, R. Tahir, S. Abdelatif, S. Boulaajaj, J.A. Mayoral, J.I. Garcia, J.M. Fraile, A. Kossir, H. Oumimoun, J. Catal. 213 (2003) 1.

[20] D.J. Macquarrie, R. Nazih, S. Sebti, Green Chem. 4 (2002) 56.

[21] D.J. Macquarrie, D.B. Jackson, Chem. Commun. (1997) 1781.

[22] J.E.G. Mdoe, J.H. Clark, D.J. Macquarrie, Syntett (1998) 625.

[23] G. Sartori, F. Bigi, R. Maggi, R. Sartorio, D.J. Macquarrie, M. Lenarda, L. Storaro, S. Coluccia, G. Martra, J. Catal. 222 (2004) 410.

[24] X.G. Wang, K.S.K. Lin, J.C.C. Chan, S. Cheng, Chem. Commun. (2004) 2762.

[25] M.C. Burleigh, M.A. Markowitz, M.S. Spector, B.P. Gaber, Chem. Mater. 13 (2001) 4760.

[26] X.S. Zhao, G.Q. Lu, A.K. Whittaker, G.J. Millar, H.Y. Zhu, J. Phys. Chem. B 101 (1997) 6525.

[27] A.S.M. Chong, X.S. Zhao, J. Phys. Chem. B 107 (2003) 12650.

[28] R.L. Shriner, C.K.F. Hermann, T.C. Morril, D.Y. Curtin, R.C. Fuson, The Systematic Identification of Organic Compounds, seventh ed., Wiley, New York, 1998.

[29] L.D. White, C.P. Tripp, J. Colloid Interface Sci. 232 (2000) 400.

[30] M. Llusar, G. Monros, C. Roux, J.L. Pozzo, C. Sanchez, J. Mater. Chem. 13 (2003) 2505.

[31] H.H.P. Yiu, P.A. Wright, N.P. Botting, J. Mol. Catal. B: Enzym. 15 (2001) 81.

[32] W. Guo, J.Y. Park, M.O. Oh, H.W. Jeong, W.J. Cho, I.K. Kim, C.S. Ha, Chem. Mater. 15 (2003) 2295.

[33] S. Sebti, A. Saber, A. Rhihil, R. Nazih, R. Tahir, Appl. Catal. A: Gen. 206 (2001) 217.

[34] F. Bigi, S. Carloni, R. Maggi, A. Mazzacani, G. Sartori, Stud. Surf. Sci. Catal. 130 (2000) 3501.

[35] L.G. Wade Jr., Organic Chemistry, Prentice-Hall, New Jersey, 1987.

[36] R. Moscovi, J.P. Ferraz, E.A. Neves, J.O. Tognoli, M.I. El Seoud, L. do Amaral, J. Org. Chem. 41 (1976) 4093. 